\title{
Dynamin-related protein I is involved in micheliolide-induced breast cancer cell death
}

\author{
This article was published in the following Dove Press journal: \\ OncoTargets and Therapy \\ 16 November 2015 \\ Number of times this article has been viewed
}

\author{
Yongsheng Jia ${ }^{1,2}$ \\ Liyan Zhou' ${ }^{1,2}$ \\ Chen Tian ${ }^{3}$ \\ Yehui Shi ${ }^{1,2}$ \\ Chen Wang ${ }^{1,2}$ \\ Zhongsheng Tong ${ }^{1,2}$ \\ 'Department of Breast Oncology, \\ Key Laboratory of Breast Cancer \\ Prevention and Therapy, Ministry of \\ Education, ${ }^{2}$ Key Laboratory of Cancer \\ Prevention and Therapy, National \\ Clinical Research Center for Cancer, \\ ${ }^{3}$ Department of Hematology, Tianjin \\ Medical University Cancer Institute \\ and Hospital, Tianjin, People's Republic \\ of China
}

\begin{abstract}
Dynamin-related protein 1 (Drp1) is a newly discovered therapeutic target for tumor initiation, migration, proliferation, and chemosensitivity. Thus, therapeutic strategies that focus on targeting Drp1 and its related signaling pathway pave a new way to address the ineffectiveness of traditional cancer therapies. Micheliolide (MCL), a guaianolide sesquiterpene lactone, can selectively eradicate acute myeloid leukemia stem or progenitor cells. But the effect of MCL on the mitochondrial dynamics of cancer cells is still not well demonstrated. In this study, we show that MCL inhibited the growth of MCF-7 human breast cancer cells, accompanied by increased mitochondrial fission and upregulation of Drp1. The results obtained from overexpression experiments of wild or dominant-negative mutant type of Drp1 demonstrate that Drp1 is both necessary and sufficient to induce MDA-MB-231 and MCF-7 cell death. Furthermore, mitochondrial membrane potential decreased, whereas reactive oxygen species (ROS) generation, cytochrome $c$ release, and PARP cleavage were enhanced after overexpression of Drp1 wild type. On the other hand, overexpression of Drp1-K38A (a dominant-negative mutant of Drp1) rescued cells from increased apoptosis, confirming the role of MCL-induced Drp1 in the observed apoptosis. Finally, MCL-induced Drp1-mediated cell death could be reversed by $\mathrm{N}$-acetyl-L-cysteine (the ROS scavenger) in breast cancer cells. Taken together, the present study shows a novel role for Drp1 in MCL-induced breast cancer cell death, potentially through regulation of ROS-mitochondrial apoptotic pathway.
\end{abstract}

Keywords: Drp1, breast cancer, apoptosis, mitochondrial dynamics

\section{Introduction}

Breast cancer, one of the most common tumors in women, is the first cause of death for malignancy in the female population. Diverse treatments have been tried for metastatic breast cancer in recent years, although chemotherapy remains the main approaches for the majority of patients. Anthracyclines and taxanes are considered to be the most effective treatment of breast cancer. However, there are two major limitations for patients with metastatic breast cancer: 1) prior exposure often leads to tumor resistance and 2) a risk of cumulative toxicities, such as cardiac damage and peripheral neuropathy. ${ }^{1}$ Therefore, novel approaches are needed. Parthenolide (PTL), a natural sesquiterpene lactone (SL) with 10,5-ring structure isolated from tanacetum parthenium, is the first small molecule found to be selectively against cancer stem cells by targeting specific signaling pathways. ${ }^{2}$ The inhibition of nuclear factor $\kappa \mathrm{B}$ and production of reactive oxygen species (ROS) appear to be important for PTL activity against cancer stem cells. ${ }^{3}$ The water-soluble Michael adduct of PTL $\left(\right.$ DMAPT) ${ }^{4}$ is now under clinical trial for cancer therapy. However, the low bioavailability of PTL is the major limitation for its use in the clinic. Micheliolide (MCL), a novel guaianolide SL derived from PTL, can selectively eliminate cancer stem cells. The dimethylamino Michael adduct of MCL (DMAMCL) showed high
Correspondence: Zhongsheng Tong Tianjin Medical University Cancer Institute and Hospital, Tiyuan North, Hexi District, Tianjin 30060, People's Republic of China

Email tongzhongsheng@tjmuch.com 
oral bioavailability. MCL/DMAMCL had at least three advantages over PTL (PTL/DMAPT): higher stability, less toxic, and more sustainable release of active drug from prodrug. ${ }^{5}$ To date, MCL and its water-soluble Michael adduct, DMAMCL, have only been reported to be used in acute myeloid leukemia ${ }^{6}$ and colorectal cancer. ${ }^{7}$ However, detailed molecular mechanisms of these anticancer effects of MCL are largely unknown.

Dynamin-related protein 1 (Drp1) is required for mitochondrial division in mammalian cells. Alterations in mitochondrial dynamics are associated with maintaining stemness properties of mammalian epithelial stem-like cells. ${ }^{8}$ Moreover, Drp1 is a newly discovered therapeutic target for tumor initiation, ${ }^{9}$ migration, ${ }^{10}$ proliferation, ${ }^{11}$ and chemosensitivity. ${ }^{12}$ In 2013 , Zhao et al ${ }^{13}$ reported that cancer cell migration and invasion were regulated by mitochondrial dynamics. Recently, Han et al reported that Drp1-dependent mitochondrial fission not only regulated hypoxia-induced migration of breast cancer cells but also facilitated its sensitivity to chemotherapeutic agents. ${ }^{14}$ Thus, targeting Drp1-dependent mitochondrial dynamics may provide a novel strategy to suppress cancer metastasis and improve the chemotherapeutic effect. The present study was designed to identify the importance of mitochondrial fission protein Drp1 in MCL-induced breast cancer cell death and to investigate the relevant mechanisms underlying the activity of Drp1 in breast cancer cells.

\section{Materials and methods Cell culture and reagents}

All human breast cancer cell line protocols were approved by the Ethics Committee Tianjin Medical University. The human breast cancer cell lines MCF-7 and MDA-MB-231 were preserved by our laboratory and cultured in Dulbecco's Modified Eagle's Medium (HyClone, Beijing, People's Republic of China), supplemented with $10 \%$ fetal bovine serum (Thermo Fisher Scientific, Waltham, MA, USA), $100 \mathrm{U} / \mathrm{mL}$ penicillin (Sigma-Aldrich Co., St Louis, MO, USA), and $100 \mu \mathrm{g} / \mathrm{mL}$ streptomycin (Sigma-Aldrich Co.). The Drp1 antibody was purchased from BD Biosciences (San Jose, CA, USA). Cytochrome $c$, poly (ADP-ribose) polymerase (PARP) antibody, and $\beta$-actin antibody were obtained from Cell Signaling Technology (MA, USA).

\section{Generation of Drpl overexpression and knockdown stable cell lines}

The Drp1 wild type (Drp1-WT) and K38A cassette were amplified using polymerase chain reaction from the Drp1-WT and K38A expression plasmid, which was graciously provided by Professor Jinning Lou, Institute of Clinical Medical Sciences, People's Republic of China - Japan Friendship Hospital (primer sequences: forward 5'-GACGAGCTGTACAAGATGGAGGCGCT AATTCCT-3' and reverse 5'-TTTAAATTCGAATTCTC TAGATCACCAAAGATGAGTCTC-3'), and the segment was inserted into a lentiviral vector using the E-Fusion Cloning Kit (Biophay Biotechnology Co, Huaian, People's Republic of China). The vector PCDH carried a puromycinresistance marker, and a green fluorescent protein was preserved by our laboratory. Lentivirus production and tittering were carried out according to the protocols from Trono Lab (Lausanne, Switzerland). ${ }^{15}$ Then, the lentivirus was resuspended in cell culture medium and incubated in wells containing breast cancer cells. The cells were treated with $1 \mathrm{mg} / \mathrm{L}$ puromycin and tested for expression of the transgene 1 week later.

\section{3-(4,5-Dimethylthiazol-2-yl)-2,5- diphenyltetrazolium bromide}

Breast cancer cells were plated at a density of $0.8 \times 10^{4}$ cells/ well in 96-well plates and treated with MCL at the indicated concentrations for 48 hours. Then, $200 \mu \mathrm{L}$ of fresh medium plus $20 \mu \mathrm{L}$ of 3-(4,5-dimethylthiazol-2-yl)2,5-diphenyltetrazolium bromide (MTT, $5 \mathrm{mg} / \mathrm{mL}$ dissolved in PBS) was added and incubated at $37^{\circ} \mathrm{C}$ for 4 hours. Finally, $100 \mu \mathrm{L}$ of DMSO was added, and the absorbance at $570 \mathrm{~nm}$ was measured using an MRX II absorbance reader (DYNEX Technologies, Chantilly, VA, USA).

\section{Western blot}

To determine the levels of protein expression, the cells were harvested, lysed in radioimmunoprecipitation assay lysis buffer (50 mM Tris $\mathrm{HCl}$ [pH 8.0], $150 \mathrm{mM} \mathrm{NaCl}$, $0.1 \%$ sodium dodecyl sulfate, $1 \%$ NP-40, $0.25 \%$ sodium deoxycholate, and $1 \mathrm{mM}$ ethylenediaminetetraacetic acid) with freshly added protease inhibitor cocktail (Hoffman-La Roche Ltd., Basel, Switzerland) on ice for 30 minutes, and subsequently centrifuged at 13,000 rpm for 10 minutes. The total protein concentration of whole-cell extracts was measured with Bradford reagent (Bio-Rad Laboratories Inc., Hercules, CA, USA). The proteins were resolved by sodium dodecyl sulfate polyacrylamide gel electrophoresis (Bio-Rad Laboratories Inc.). After electrophoresis, the proteins were electrotransferred to polyvinylidene fluoride membranes (EMD Millipore, Billerica, MA, USA), blocked with 5\% skimmed milk, and incubated with antibodies. The immune complexes were detected using the LI-COR Odyssey CLx Infrared Imaging System (LI-COR, Lincoln, NE, USA). 


\section{Confocal microscopy}

Cells treated with or without $10 \mu \mathrm{M}$ MCL for 24 hours at $37^{\circ} \mathrm{C}$ were incubated with $1 \mu \mathrm{M}$ MitoTracker Red (Molecular Probes, Thermo Fisher Scientific, Waltham, MA, USA) at $37^{\circ} \mathrm{C}$ for 15 minutes. After fixed with $4 \%$ formaldehyde for 15 minutes at room temperature, the mitochondrial morphology was viewed under a confocal microscope (Olympus IX81; Olympus Corporation, Tokyo, Japan).

\section{Observation of morphologic changes}

The $3 \times 10^{5} /$ well breast cancer cells were cultured in sixwell plates and treated with or without MCL for 24 hours. Then cell morphology was observed using a Nikon Eclipse TE2000-U inverted microscope coupled with a DigitalSight DS-2Mv digital camera (Nikon Corporation, Tokyo, Japan).

\section{Apoptosis analysis}

Cells were cultured at a concentration of $3 \times 10^{5} /$ well in sixwell plates and then treated with or without $10 \mu \mathrm{M}$ MCL for 24 hours. After that, $5 \mu \mathrm{L}$ Annexin V-PE and $5 \mu \mathrm{L}$ 7-AAD (BD Biosciences) were added according to the manufacturer's instruction and analyzed using fluorescence-activated cell sorting within 1 hour.

\section{Mitochondrial membrane potential}

Mitochondrial membrane potential $(\Delta \Psi m)$ indicates that the initiation of cell apoptosis was quantified by staining with the cationic dye JC-1 (Sigma-Aldrich Co.). JC-1 accumulates in the mitochondria of healthy cells and fluoresces red $(560 \mathrm{~nm})$. When the $\Delta \Psi m$ collapses, JC-1 uptake is limited to the cytoplasm where it fluoresces green $(530 \mathrm{~nm})$. Cells were treated with or without $10 \mu \mathrm{M}$ MCL for 24 hours. Afterward, the cells were stained with JC-1 $(10 \mu \mathrm{g} / \mathrm{mL})$ for 20 minutes in the dark at $37^{\circ} \mathrm{C}$. Stained cells were rinsed twice with JC-1 staining buffer and then analyzed by the microplate reader of Tecan Infinite M200 (Tecan, Austria). The green/red fluorescence:intensity ratio was calculated as $\Delta \Psi m$.

\section{Flow cytometric analysis of intracellular ROS production}

Cells were cultured at a concentration of $3 \times 10^{5} /$ well in sixwell plates and then treated with or without $10 \mu \mathrm{M}$ MCL for 4 hours. Then, the cells were incubated with $5 \mu \mathrm{M}$ MitoSOX RED (Molecular Probes, Thermo Fisher Scientific) at $37^{\circ} \mathrm{C}$ for 10 minutes. ${ }^{16}$ Samples were analyzed at an excitation wavelength of $510 \mathrm{~nm}$ and an emission wavelength of $580 \mathrm{~nm}$ using a FACScan flow cytometer (Becton-Dickinson, NJ, USA).

\section{Statistical analysis}

All experiments were performed at least three times. Excel (Microsoft Corporation, Redmond, WA, USA) was used for statistical analysis, and statistical significance was determined using Student's $t$-test. $P<0.05$ was considered significant.

\section{Results \\ MCL-inhibited human breast cancer cell growth in vitro}

The compounds of PTL and MCL contain $\alpha, \beta$-unsaturated carbonyl structures, including SLs and their derivatives, as shown in Figure 1A. Various concentrations $(0-40 \mu \mathrm{M})$ of MCL and PTL were added to MCF-7 cells for 48 hours and then determined by MTT assay (Figure 1B). Results showed that MCL and PTL significantly decreased cell viability in a dose-dependent manner. The half-maximal inhibitory concentration value of PTL $(27.7 \mu \mathrm{M})$ was higher than that of MCL $(21.8 \mu \mathrm{M}), P<0.05$. Similar results were observed in MDA-MB-231 cells after treatment with MCL or PTL (Figure 1C).

\section{MCL-induced mitochondrial}

\section{fragmentation and upregulation of Drp I}

To determine the effect of MCL on Drp1 expression, Western blot was done and showed that Drp1 was upregulated in a dose-dependent manner in MCF-7 cells (Figure 2A) and MDA-MB-231 cells (Figure 2B). Furthermore, the confocal microscope showed that mitochondria were changed from long shape to round and short shape after treatment (Figure 2C), suggesting mitochondrial fission.

\section{Characterization of Drpl-WT and Drpl- $\mathrm{K} 38 \mathrm{~A}$ cell lines}

Point mutations (Figure 3A) in the guanosine- 5 '-triphosphate (GTP)-binding domain of Drp1 (K38A) dramatically changed its intracellular distribution from a punctate vesicular structures to an aggregated pattern (Figure 3B). To further investigate the implication of Drp1 in breast cancer cells, we established two stable cell lines with Drp1-WT or Drp1K38A overexpression, respectively. The transgene-encoded protein levels were analyzed by Western blot. As shown in Figure 3C, Drp1-WT and Drp1-K38A show high-level induction and lowest background.

\section{Overexpression of Drpl induced decreased survival of breast cancer cells in response to $\mathrm{MCL}$}

After treatment, MTT assay was used to examine the cytotoxic effects of MCL against MCF-7 (Figure 4A) and 
A<smiles>C=C1C(=O)O[C@H](CC)[C@@H]1CCC1=CCC[C@]2(C)O[C@@H]12</smiles>

PTL<smiles>C=C1C(=O)O[C@H]2[C@H]1CCC(C)=C1CC[C@](C)(O)[C@H]12</smiles>

$\mathrm{MCL}$
B

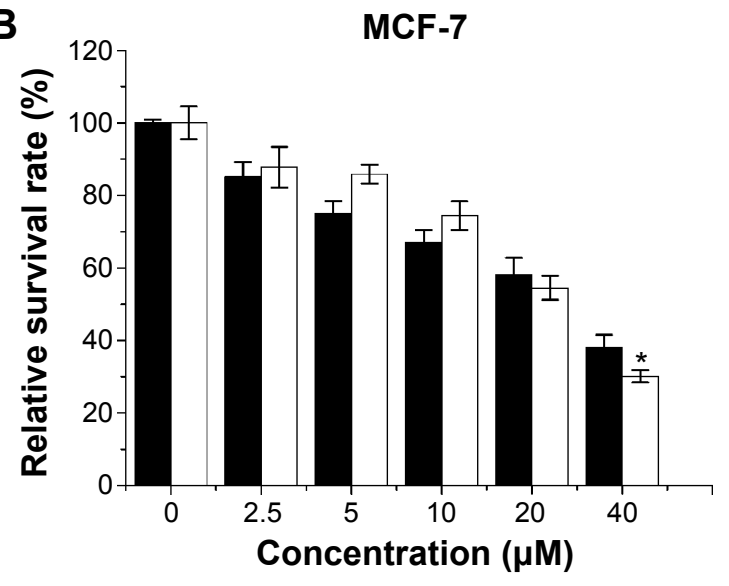

C

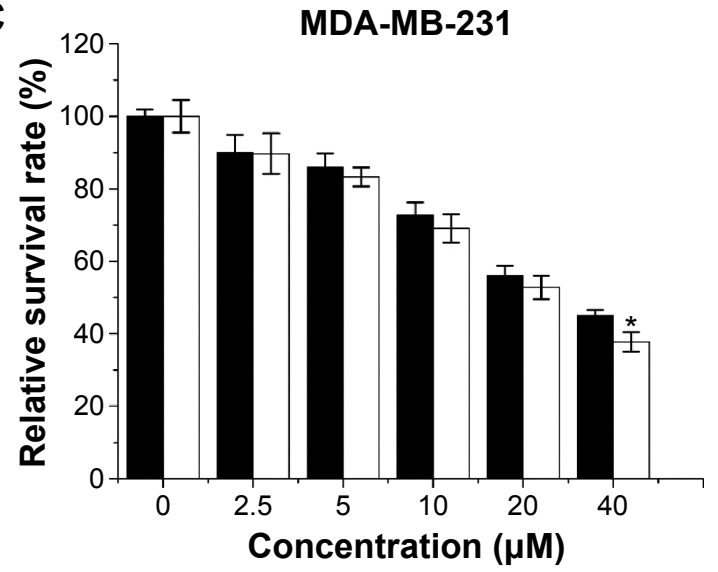

PTL $\square$ MCL

Figure I MCL-inhibited human breast cancer cell growth in vitro.

Notes: (A) Structures of MCL and PTL. (B) Comparison between the cytotoxicity of MCL and PTL to MCF-7 cells by MTT assay. (C) Comparison between the cytotoxicity of MCL and PTL to MDA-MB-23I cells by MTT assay. Data are presented as the mean $\pm S D(n=3)$. $* P<0.05$.

Abbreviations: MCL, micheliolide; PTL, parthenolide; MTT, 3-(4,5-dimethylthiazol-2-yl)-2,5-diphenyltetrazolium bromide; SD, standard deviation.

A

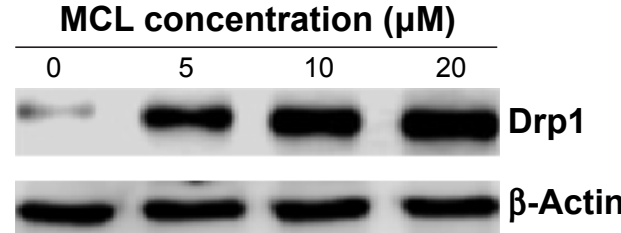

C

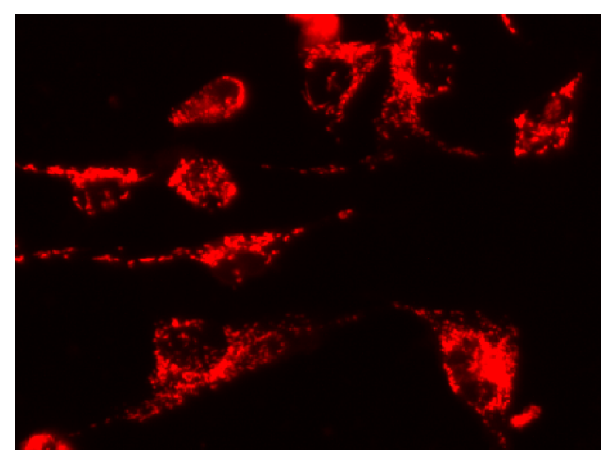

B

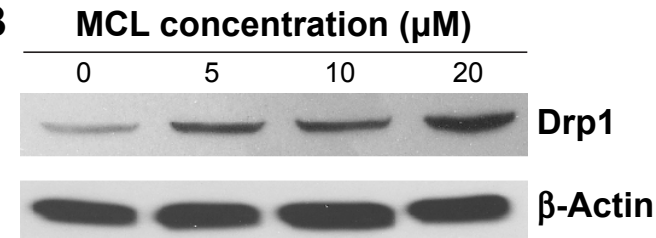

MCL $(10 \mu M)$

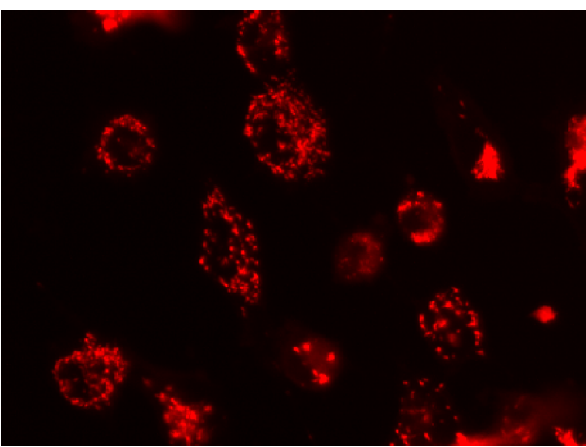

Figure $2 \mathrm{MCL}$-induced mitochondrial fragmentation and upregulation of Drpl protein.

Notes: Dose-response of MCL on Drpl expression in (A) MCF-7 cells and (B) MDA-MB-23I cells was analyzed by Western blot. (C) Confocal microscope (400x) for mitochondrial morphology in MCF-7 cells was treated with or without $10 \mu \mathrm{M}$ MCL for 24 hours.

Abbreviations: MCL, micheliolide; DrpI, dynamin-related protein I. 


\section{A Drp1-WT}

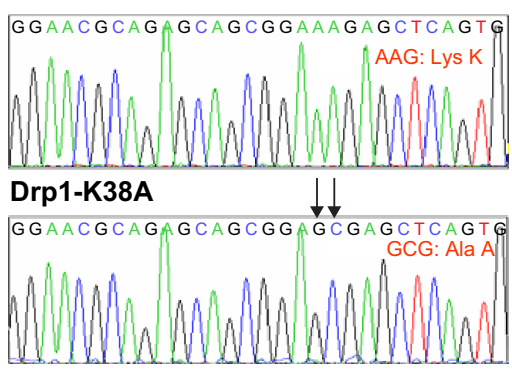

C

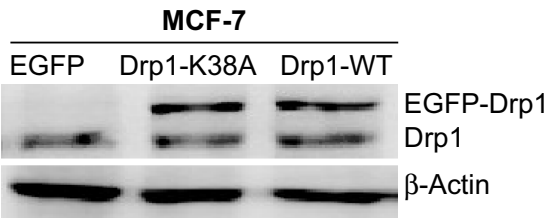

D

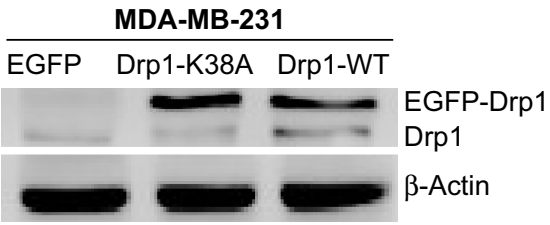

\section{B PCDH-P-EGFP-DrP1-K38A}
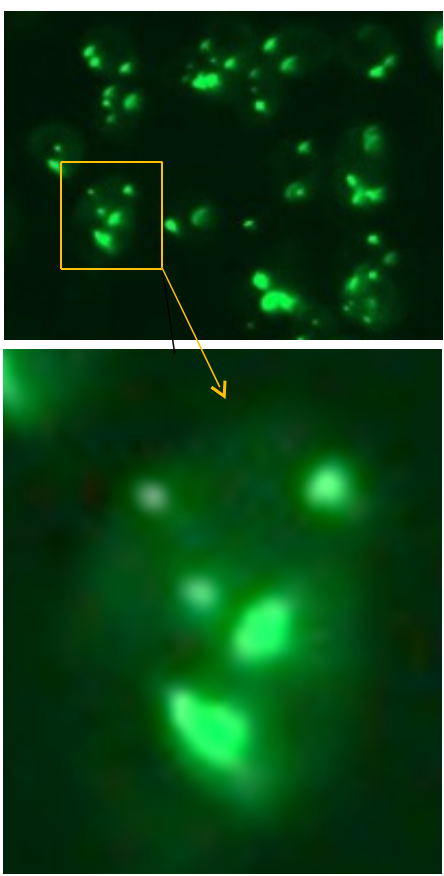

PCDH-P-EGFP-Drp1-WT
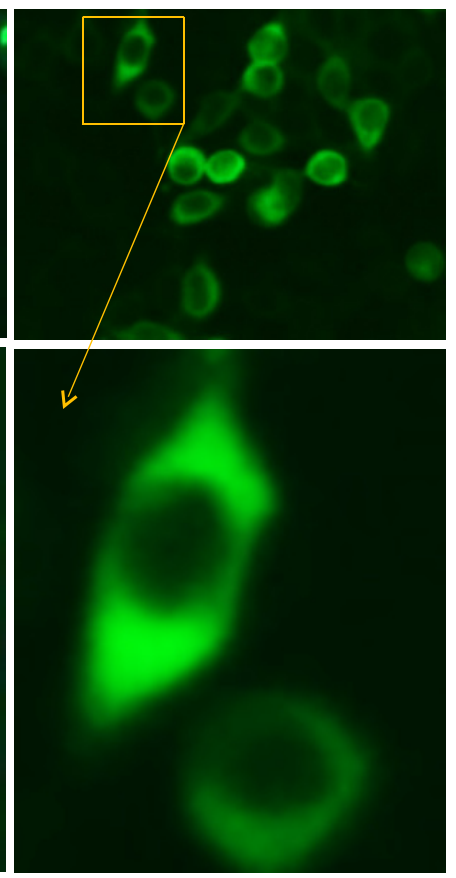

Figure 3 Characterization of Drpl-WT and Drpl-K38A cell lines.

Notes: (A) Full-length, untagged DrpI-WT and K38A products were subcloned into PCDH-EGFP and sequenced on an automated DNA sequencer, which results from a 2-bp alteration (AAG $\rightarrow$ GCG) leading to a substitution of lysine to alanine in codon 38. (B) EGFP-tagged WT, K38A shows different localizations. HEK293T cells transfected with PCDH-EGFP-DrpI-WT and PCDH-EGFP-DrpI-K38A plasmids were observed by fluorescent microscope (200×). EGFP-tagged DrpI-WT distributes to punctate vesicle-like structures similar to the distribution described for endogenous DrpI. In contrast, K38A accumulates into large aggregates and also smaller punctuate foci similar to those observed in the WT cells. DrpI protein expressions in (C) MCF-7 and (D) MDA-MB-23I cells were detected by Western blot. $\beta$-Actin was used as loading control. Abbreviations: DrpI, dynamin-related protein I; WT, wild type; EGFP, enhanced green fluorescent protein.

MDA-MB-231 cells (Figure 4B). Results showed that cell viability was decreased in a dose-dependent manner after treatment. The half-maximal inhibitory concentration value of MCL was reduced from 22.9 to $16.6 \mu \mathrm{M}$ in MCF-7 cells and from 23.3 to $14.8 \mu \mathrm{M}$ in MDA-MB-231 cells, respectively, after transduction of Drp1-WT. The most obvious sensitivity effect was observed at the concentration of $10 \mu \mathrm{M}$ for both cell lines, which suggested that breast cancer cells with Drp1 overexpression were more sensitive to MCL than their parental and Drp1-K38A counterparts.
A

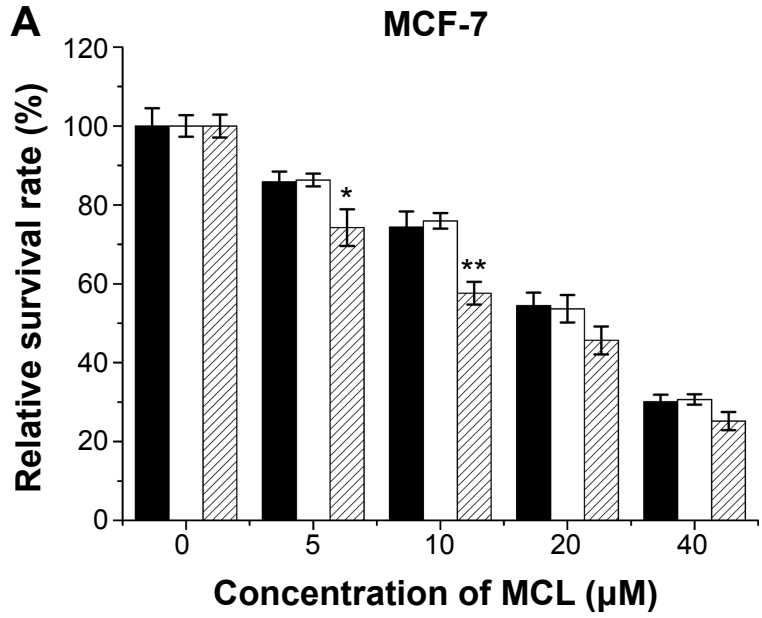

B

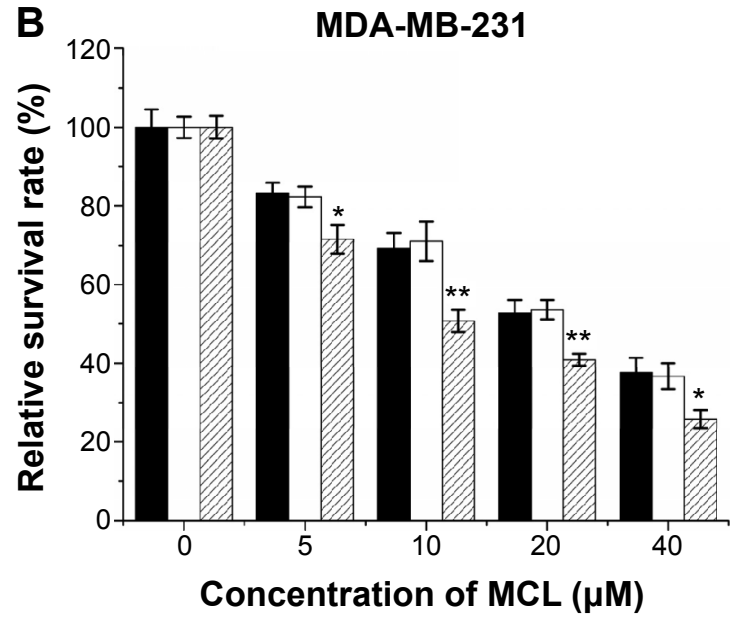

EGFP $\square$ Drp1-K38A VIIIA Drp1-WT

Figure 4 Overexpression of Drpl induces decreased survival of breast cancer cells in response to MCL.

Notes: Cell viability was determined by MTT assay. The cell survival rates were calculated by comparing with the control group (I00\%) after 48 hours treatment. Data are presented as the mean \pm SD ( $n=3)$. (A) MCF-7 cells. (B) MDA-MB-23I cells. $* p<0.05 ; * * p<0.01$

Abbreviations: DrpI, dynamin-related protein I; MCL, micheliolide; MTT, 3-(4,5-dimethylthiazol-2-yl)-2,5-diphenyltetrazolium bromide; SD, standard deviation; WT, wild type. 


\section{Drpl overexpression enhanced breast cancer cells apoptosis induced by MCL}

To explore whether Drp1-WT breast cancer cells were more sensitive to MCL-induced apoptosis than control cells, we compared morphological changes among control, Drp1-K38A, and Drp1-WT breast cancer cells incubated with various concentrations of MCL for 24 hours. Results showed that after treatment, more Drp1-WT breast cancer cells exhibited distinctly shrinked and rounded shapes compared with control cells. In addition, apoptosis results showed that the apoptosis rate of MCF-7 and MDA-MB231 cells were increased after MCL treatment. Similarly, the apoptosis rate of MCF-7 and MDA-MB-231 cells with Drp1 overexpression was enhanced (Figure 5A), which indicated that MCF-7 and MDA-MB-231 cells with high Drp1 levels were more sensitive to MCL-induced cell death.

\section{Overexpression of Drpl decreased mitochondrial membrane potential after MCL treatment}

The $\Delta \Psi m$ was measured by flow cytometry using the potential-sensitive probe JC-1. As shown in Figure 6, overexpression of Drp1-WT led to $\Delta \Psi m$ depolarization in breast cells cultured with MCL. Moreover, overexpression of Drp1-WT resulted in increased release of mitochondrial cytochrome $c$ in MCF-7 cells (Figure 7) and enhanced with MCL treatment. In contrast, opposite results were observed in Drp1-K38A cells (Figures 6 and 7), which were consistent with the reports that mitochondria eventually depolarize during the release of cytochrome $c$ and the activation of the caspase cascade. ${ }^{17}$ Furthermore, PARP cleavage, an apoptosis-related protein, was expressed after MCL treatment in Drp1-overexpressed cells (Figure 7).

\section{Drpl overexpression expanded superoxide generation after $\mathrm{MCL}$ treatment}

As mentioned previously, oxidative stress has been associated with MCL-induced cell damage. Here, we assessed whether ROS level in MCL-treated breast cancer cells could be regulated by Drp1. First, we compared the ROS levels in enhanced green fluorescent protein (EGFP), Drp1-K38A, and Drp1-WT breast cells after addition of MCL (Figure 8A and B). Results showed that ROS level in Drp1 cells was higher compared with EGFP and Drp1-K38A cells, which indicated that overexpression of Drp1 might potentiate MCLinduced ROS generation following cell damage with the MCL concentration of $10 \mu \mathrm{M}$. N-Acetyl-L-cysteine (NAC), the ROS scavenger, is commonly used to identify and test ROS inducers and to inhibit ROS. Thus, NAC was pretreated before MCL administration to estimate the effect of ROS in the Drp1-induced MCL sensitivity of breast cancer cells. Oxidative stress mediates apoptotic changes were detected after MCL treatment in MCF-7 and MDA-MB-231 cells. In contrast, after pretreatment with NAC, the morphological alterations in apoptotic cell death which concern both membrane blebbing and granular apoptotic bodies were obviously attenuated (Figure 8C). The cell viability assay also indicated that NAC pretreatment dramatically lessen the proliferation inhibition ratio of EGFP, Drp1-K38A, and Drp1-WT MCF-7 cells (Figure 8D) from 25.6\%, 24.1\%, and 42.37\% (10 $\mu \mathrm{M}$ of MCL alone) to $12 \%, 15 \%$, and $16 \%$ (in the presence of $5 \mathrm{mM}$ of NAC), respectively. MDA-MB-231 cells displayed a similar pattern that observed in MCF-7 cells (Figure 8E). There was markedly no difference between the MCL sensitivity of the EGFP, Drp1-K38A, and Drp1-WT breast cancer cells and was detected after cotreatment with NAC, which indicated that ROS is a critical determinant of MCL toxicity in breast cancer cells during Drp1 overexpression.

\section{Discussion and conclusion}

In the past years, mitochondrial dynamics gained increasing interest. An area of research beyond Warburg is emerging on the mechanistic understanding of how mitochondrial dynamics disorders contributes to cell metabolism, proliferation, and tumorigenesis. ${ }^{18}$ Targeting these key proteins of the fission or fusion mitochondrial machinery can be a promising therapeutic strategy for oncotherapy. Signaling pathways underlying mitochondrial dynamics will shed a light on clinical management of cancer.

The mitochondrion is a membrane-bound organelle essentially required for supplying cellular energy, cellular differentiation, cell death, the control of the cell cycle, and cell growth. ${ }^{19}$ Mitochondrial morphology, which is important for the maintenance of cell function and survival, is regulated by the delicate balance between fission and fusion. In mammals, two GTPases of the outer membrane named mitofusins 1 and 2 (Mfn1 and Mfn2) coordinately regulate mitochondrial fusion. ${ }^{20}$ Proteins controlling mitochondrial fission include Drp1 and Fis1. Drp1 is a cytosolic GTPase, which oligomerizes at the fission site and hydrolyzes GTP to drive membrane ingression. The profission mitochondrial protein Fis1 is an outer membrane receptors of Drp1, which is proposed to act in the recruitment and association of Drp1 with mitochondria and is thus considered as the limiting factor in the fission process. ${ }^{21}$ 
In this study, we found that MCL upregulated Drp1 expression and induced breast cancer cells apoptosis, indicating that apoptosis and mitochondrial fission changes are linked processes in breast cancer cells after MCL treatment. In order to confirm our prediction, lentiviral transduction was used to create stable breast cancer cell lines, which have incorporated the vector and express Drp1-WT and dominant-negative mutant Drp1-K38A, respectively. We found that induction of Drp1-WT was sufficient to promote apoptosis in breast cancer cells after MCL treatment, whereas
A

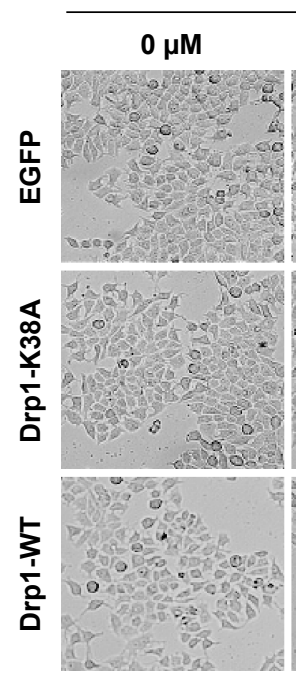

MCL (MCF-7)
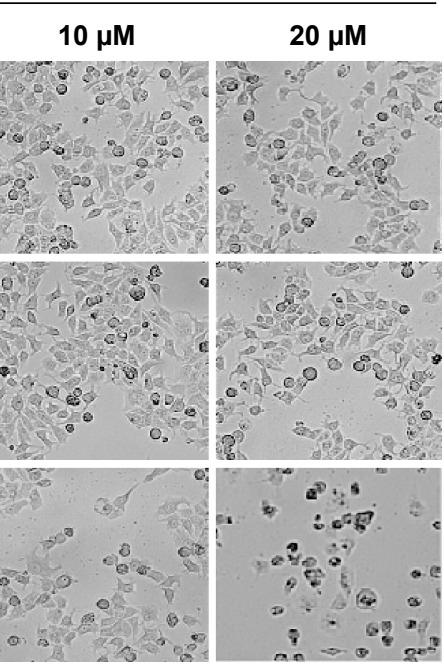

C
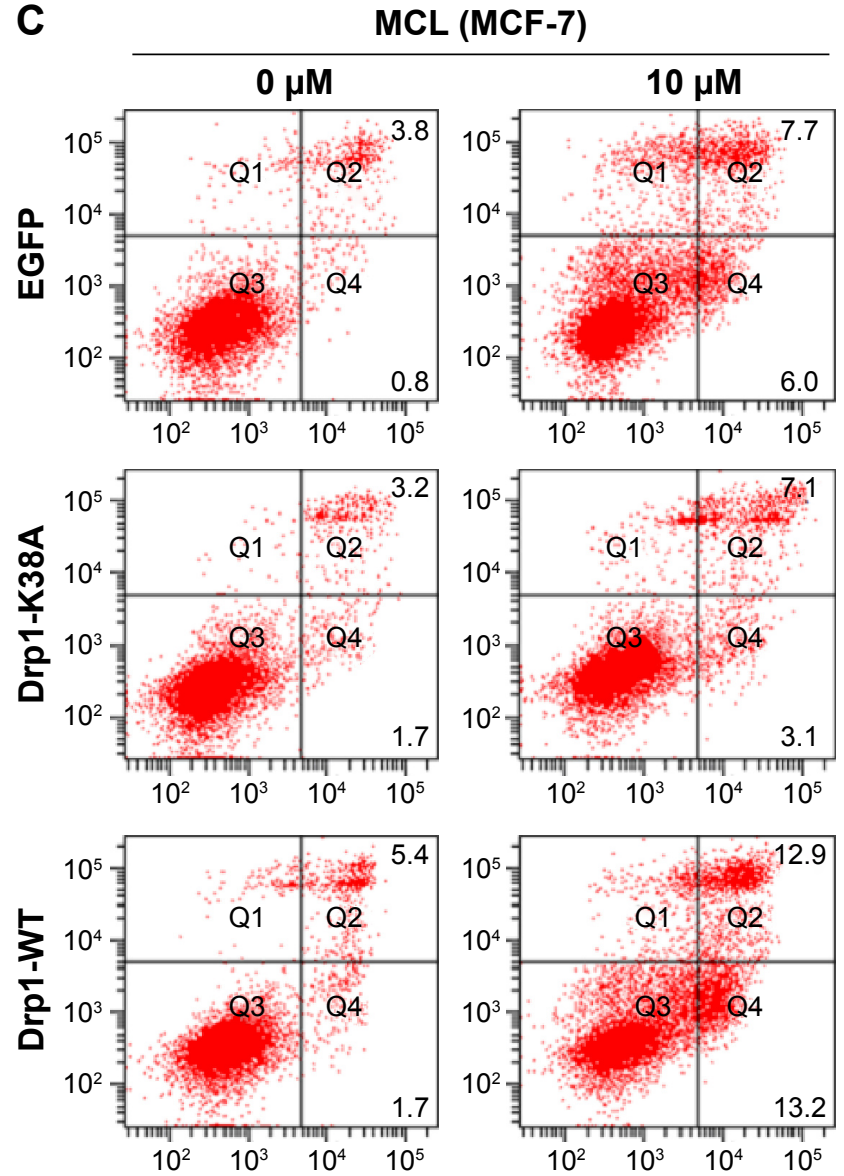

B

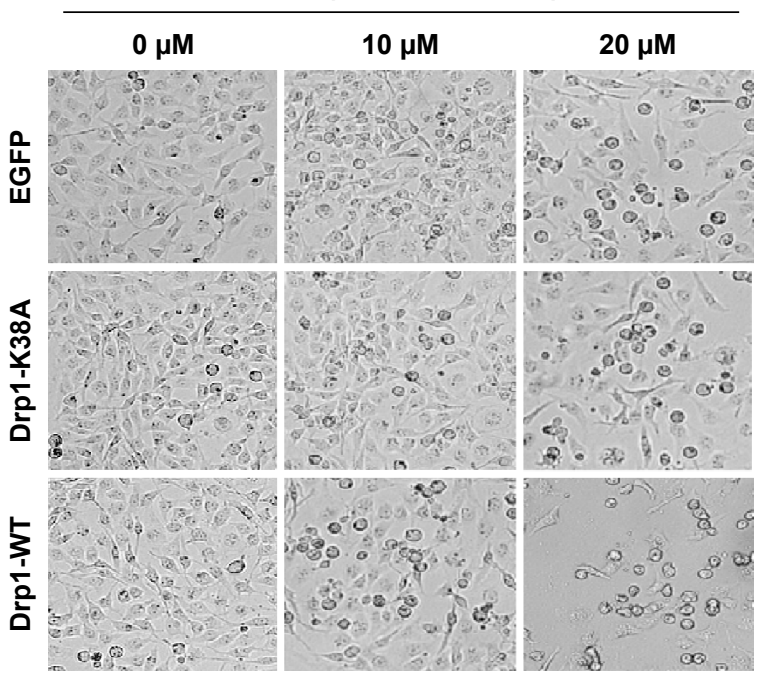

MCL (MDA-MB-231)

\section{MCF-7}

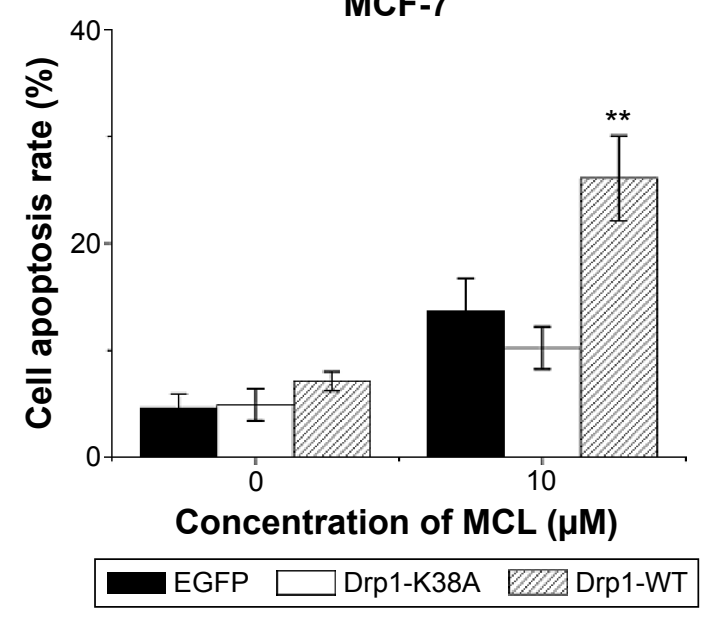

Figure 5 (Continued) 
D

MCL (MDA-MB-231)
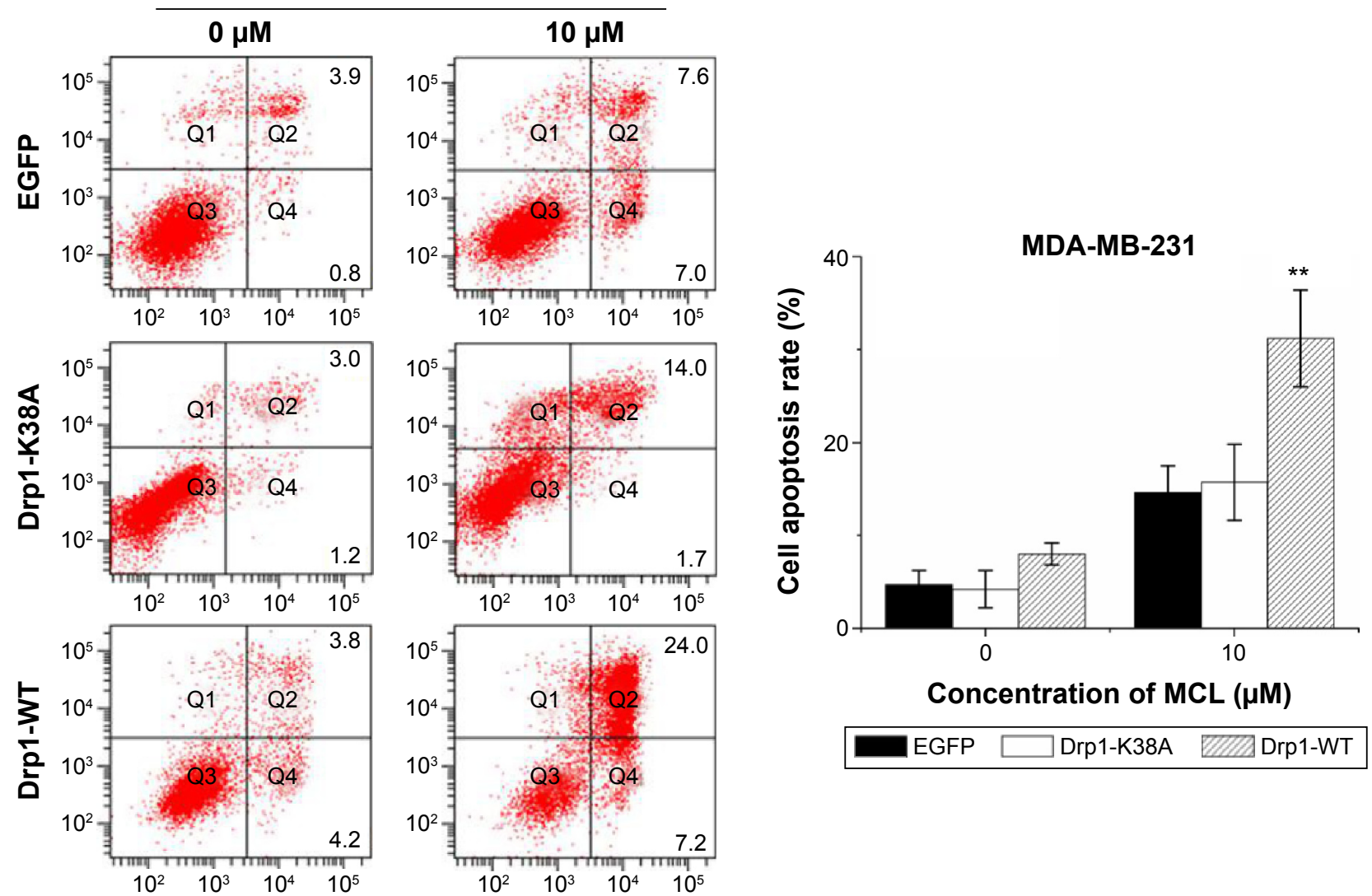

Concentration of MCL $(\mu \mathrm{M})$

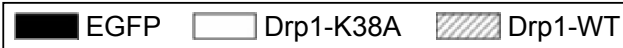

Figure 5 Drpl overexpression sensitizes breast cancer cells to MCL-induced apoptosis.

Notes: The breast cancer cells were treated with $0 \mu \mathrm{M}, 10 \mu \mathrm{M}$, and $20 \mu \mathrm{M} \mathrm{MCL}$ for 24 hours; the cellular morphologic changes were observed by phase contrast microscope (200× magnification). (A) MCF-7 cells. (B) MDA-MB-23I cells. The breast cancer cells were treated with or without $10 \mu M$ MCL for 24 hours, stained by phosphatidylserine exposure with Annexin V-PE antibody and analyzed by FACS. Combined results of three separate FACS analysis depicting the mean levels of apoptotic cells (Annexin $\mathrm{V}+$ ). Data presented as the mean \pm SD $(n=3)$. (C) MCF-7 cells. (D) MDA-MB-23I cells. $* * P<0.01$.

Abbreviations: Drpl, dynamin-related protein I; WT, wild type; EGFP, enhanced green fluorescent protein; MCL, micheliolide; FACS, fluorescence activated cell sorting; SD, standard deviation.

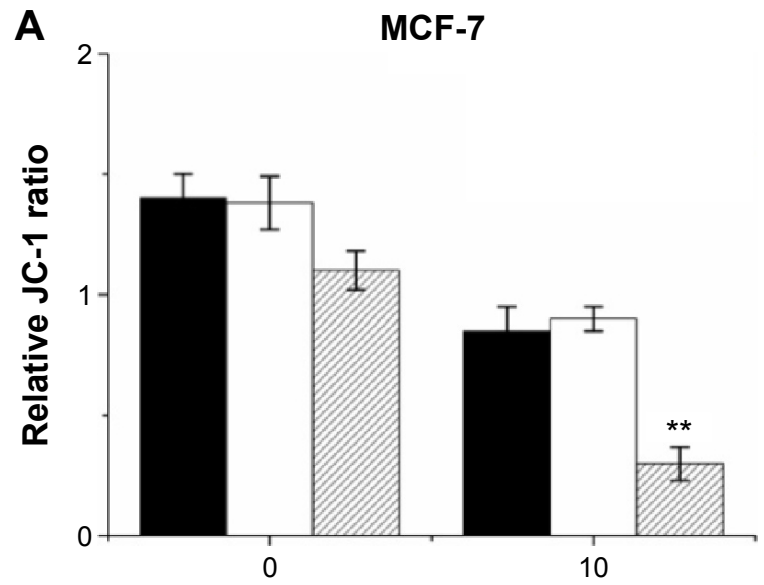

Concentration of MCL $(\mu \mathrm{M})$

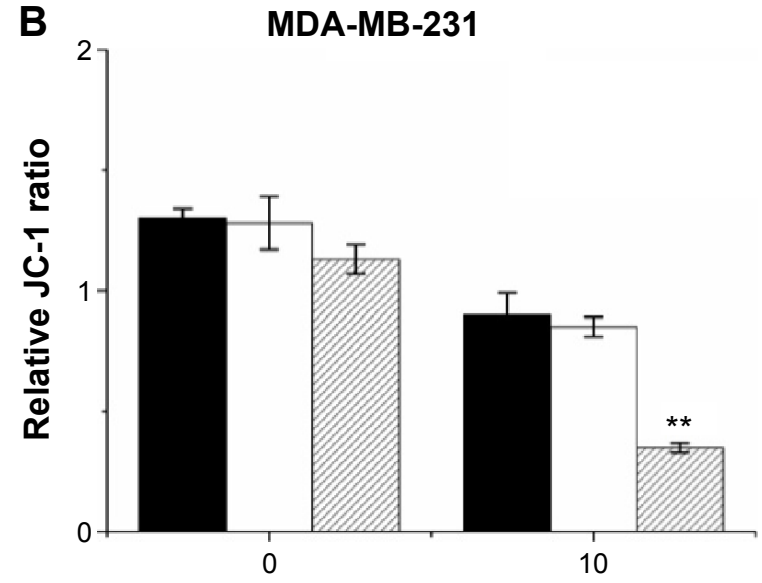

Concentration of MCL $(\mu \mathrm{M})$

\section{EGFP $\square$ Drp1-K38A WDrp1-WT}

Figure 6 Effect of Drpl on mitochondrial membrane potential.

Notes: Effect of Drpl overexpression on mitochondrial transmembrane potential $(\Delta \Psi m)$ in breast cancer cells. (A) MCF-7 cells. (B) MDA-MB-23I cells. Data presented as mean $\pm S D(n=3)$. $* * p<0.01$.

Abbreviations: DrpI, dynamin-related protein I; MCL, micheliolide; SD, standard deviation; WT, wild type. 
A
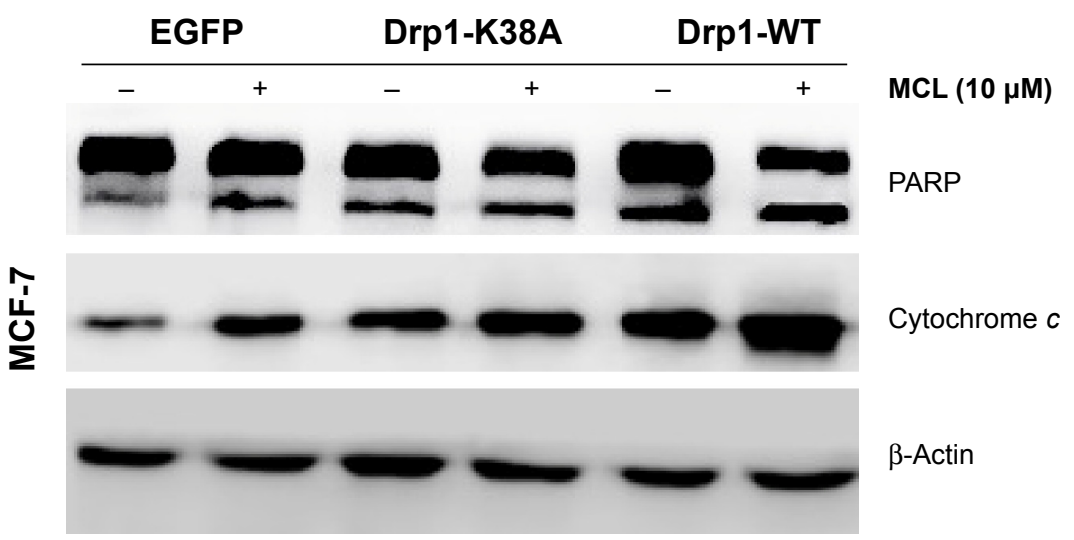

B

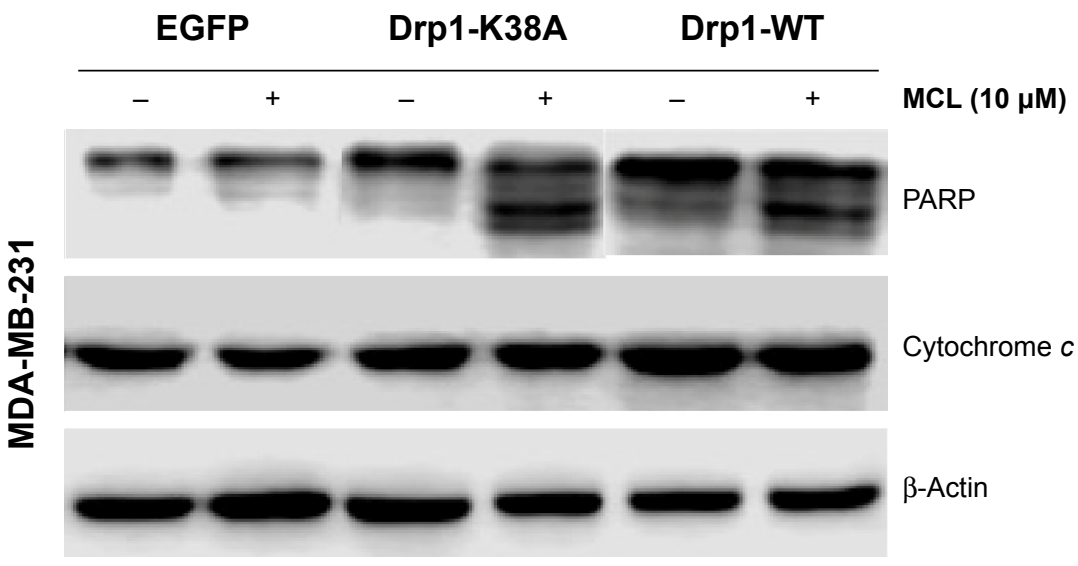

Figure 7 Effect of DrpI on the release of cytochrome $c$ from mitochondria and PARP cleavage.

Notes: The breast cancer cells were treated with or without $10 \mu \mathrm{M} M C L$ for 24 hours, the release of cytochrome $c$ from mitochondria and PARP cleavage in EGFP, DrpI-WT, and DrpI-K38A cells were detected by Western blot. (A) MCF-7 cells. (B) MDA-MB-23I cells.

Abbreviations: Drpl, dynamin-related protein I; MCL, micheliolide; PARP, poly(ADP-ribose) polymerase; SD, standard deviation; WT, wild type; EGFP, enhanced green fluorescent protein.

no apparent apoptotic effects were observed in Drp1-K38A breast cancer cells.

First, we observed that breast cancer cells display altered mitochondrial structure after MCL treatment, disintegration of tubular-shaped networks of interconnected organelles into smaller, fragmented mitochondria. Accordingly, the increasing numbers of mitochondrial division were also detected in Drp1-WT overexpression breast cancer cells. In addition, synergistic effects of Drp1-WT and MCL were clearly demonstrated. In contrast, Drp1-K38A, the dominant-negative mutant form of the mitochondrial fission protein Drp1, induces mitochondrial fusion and confers resistance to apoptosis by MCL. Therefore, we can infer that Drp1 served as a key regulator in MCL-induced mitochondrial fission and ensuing apoptosis in breast cancer cells.

Second, we identified the related signaling pathways underlying MCL-induced mitochondrial fission and apoptosis. Exogenously, ROS generation stress has been shown to selectively kill tumor cells; low levels of ROS are associated with the stemness of stem cells and cancer stem cells. ${ }^{22}$ Indeed, augmenting oxidative stress is a central antitumor mechanism of PTL. ${ }^{24,25}$ Röth et al $^{23}$ showed that activation-induced ROS generation was dependent on mitochondrial fission. Consequently, MCL must affect other pathways relevant to cancer cell death. One such pathway appears to be mediated by the activity of Drp1. As an important parameter of mitochondrial function, $\Delta \Psi m$ is maintaining the respiratory chain to generate adenosine triphosphate. A significant loss of $\Delta \Psi m$ renders cells depleted of energy with subsequent cytochrome $c$ release, following proapoptosis protein activation and cell death. In our study, the MCL-induced cell apoptosis pathway was reinforced after overexpression of Drp1-WT. In contrast, under the same conditions, induction of Drp1-K38A efficiently reduced the apoptosis, promoting effect of MCL.

Taken together, our findings identify a feed-forward mechanism whereby early Drp1 upregulation under MCL treatment, resulting in mitochondrial fission and increased ROS generation, loss of mitochondrial membrane potential, augmented cytochrome $c$ release that induces PARP cleavage, and amplifies the apoptotic signal in breast cancer cells. 
A

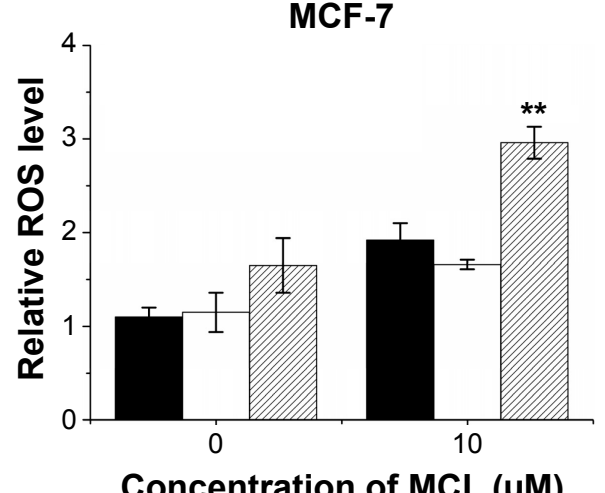

B

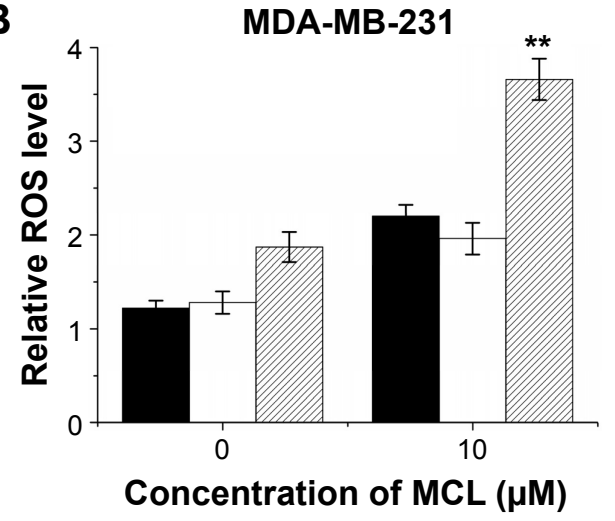

EGFP $\square$ Drp1-K38A TIm Drp1-WT

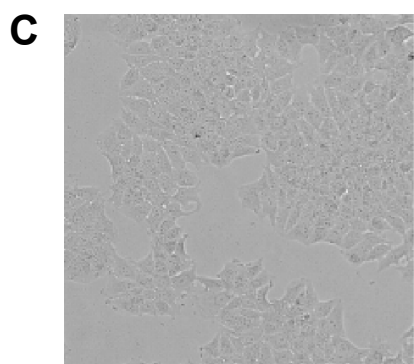

Control

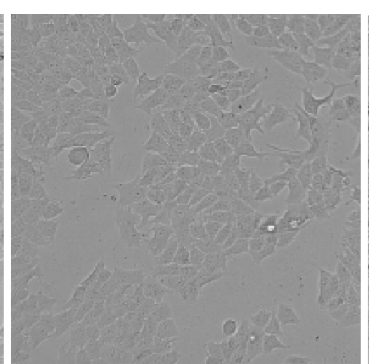

NAC

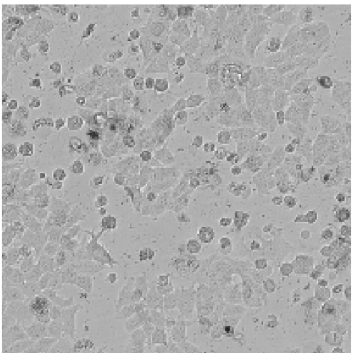

MCL

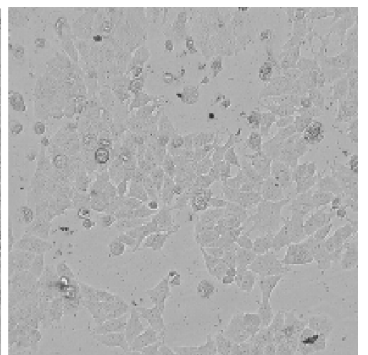

MCL + NAC

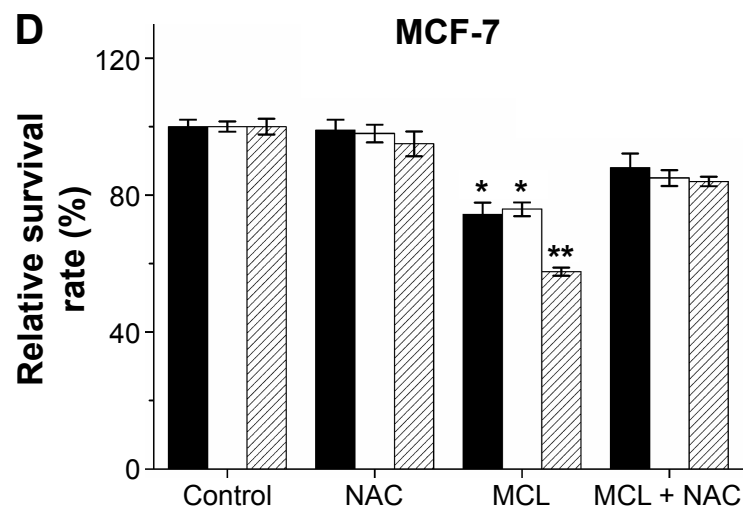

E

MDA-MB-231

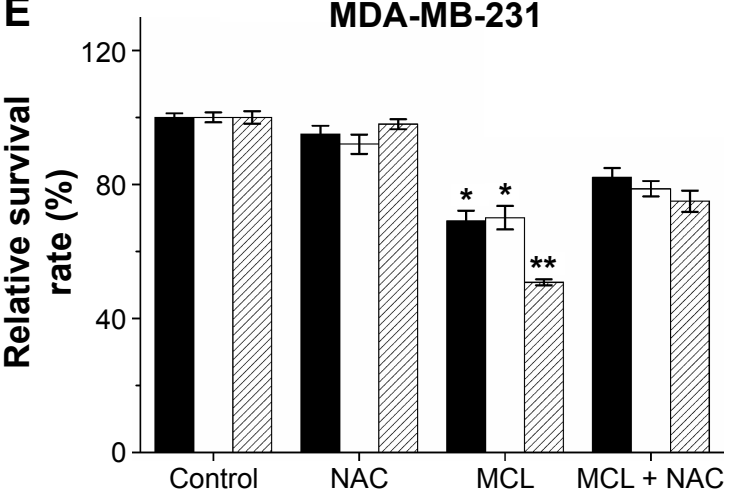

EGFP

Drp1-K38A VIIA Drp1-WT

Figure 8 Overexpression of Drpl expanded superoxide generation after MCL treatment.

Notes: The cells were treated with or without $10 \mu \mathrm{M} M C L$ for 4 hours. The MitoSOX-positive cells were measured by flow cytometry. The relative ROS level was defined as the measured ROS level relative to the control cells, which was set to I. (A) MCF-7 cells. (B) MDA-MB-23I cells. The cells were treated with or without I0 $\mu$ M MCL for 48 hours in the presence or absence of $5 \mathrm{mM}$ NAC. (C) The cellular morphologic changes were observed by phase contrast microscope (400 $\times$ magnification) in MCF-7 cells. The cell growth inhibitory ratio was measured by an MTT assay. (D) MCF-7 cells. (E) MDA-MB-23I cells. Data presented as the mean \pm SD ( $n=3$ ). $* P<0.05$; $* * P<0.01$. Abbreviations: DrpI, dynamin-related protein I; MCL, micheliolide; ROS, reactive oxygen species; NAC, N-Acetyl-L-cysteine, SD, standard deviation; WT, wild type.

\section{Conclusion}

In summary, our study showed that increased Drp1 is involved in MCL-induced breast cancer cell death via the ROS-mitochondrial apoptotic pathway. These findings will potentially be utilized in breast cancer therapy.

\section{Acknowledgments}

This work was supported by grants from National Science and Technology Pillar Program (2015BAI12B15), National Nature Science Foundation of China (81302080 and 31301161), Anticancer Key Technologies R\&D 
Program of Tianjin (12ZCDZSY16200), Research Fund for the Doctoral Program of Higher Education of China (20131202120003), Natural Science Foundation of Tianjin (14JCQNJC11100), and Foundation of Tianjin Medical University (2013KYQ06).

\section{Author contributions}

All authors made substantial contributions to conception and design, acquisition of data, or analysis and interpretation of data, took part in either drafting the article or revising it critically for important intellectual content, gave final approval of the version to be published, and agree to be accountable for all aspects of the work.

\section{Disclosure}

The authors report no conflicts of interest in this work.

\section{References}

1. Doherty MK, Morris PG. Eribulin for the treatment of metastatic breast cancer: an update on its safety and efficacy. Int J Womens Health. 2015; $7: 47-58$.

2. Ghantous A, Sinjab A, Herceg Z, Darwiche N. Parthenolide: from plant shoots to cancer roots. Drug Discov Today. 2013;18:894-905.

3. Liu Y, Lu WL, Guo J, et al. A potential target associated with both cancer and cancer stem cells: a combination therapy for eradication of breast cancer using vinorelbine stealthy liposomes plus parthenolide stealthy liposomes. J Control Release. 2008;129:18-25.

4. Neelakantan S, Nasim S, Guzman ML, Jordan CT, Crooks PA Aminoparthenolides as novel anti-leukemic agents: discovery of the NF-kappaB inhibitor, DMAPT (LC-1). Bioorg Med Chem Lett. 2009; 19:4346-4349.

5. Zhang Q, Lu Y, Ding Y, et al. Guaianolide sesquiterpene lactones, a source to discover agents that selectively inhibit acute myelogenous leukemia stem and progenitor cells. J Med Chem. 2012;55:8757-8769.

6. Ding YH, Fan HX, Long J, Zhang Q, Chen Y. The application of heck reaction in the synthesis of guaianolide sesquiterpene lactones derivatives selectively inhibiting resistant acute leukemic cells. Bioorg Med Chem Lett. 2013;23:6087-6092.

7. Viennois E, Xiao B, Ayyadurai S, et al. Micheliolide, a new sesquiterpene lactone that inhibits intestinal inflammation and colitis-associated cancer. Lab Invest. 2014;94:950-965.

8. Katajisto P, Dohla J, Chaffer CL, et al. Stem cells. Asymmetric apportioning of aged mitochondria between daughter cells is required for stemness. Science. 2015;348:340-343.

9. Xie Q, Wu Q, Horbinski CM, et al. Mitochondrial control by DRP1 in brain tumor initiating cells. Nat Neurosci. 2015;18:501-510.

OncoTargets and Therapy

\section{Publish your work in this journal}

OncoTargets and Therapy is an international, peer-reviewed, open access journal focusing on the pathological basis of all cancers, potential targets for therapy and treatment protocols employed to improve the management of cancer patients. The journal also focuses on the impact of management programs and new therapeutic agents and protocols on

Submit your manuscript here: http://www.dovepress.com/oncotargets-and-therapy-journal
10. Ferreira-da-Silva A, Valacca C, Rios E, et al. Mitochondrial dynamics protein drp1 is overexpressed in oncocytic thyroid tumors and regulates cancer cell migration. PLoS One. 2015;10:e0122308.

11. Qian W, Choi S, Gibson GA, Watkins SC, Bakkenist CJ, Van Houten B. Mitochondrial hyperfusion induced by loss of the fission protein Drp1 causes ATM-dependent G2/M arrest and aneuploidy through DNA replication stress. J Cell Sci. 2012;125:5745-5757.

12. Wang JX, Li Q, Li PF. Apoptosis repressor with caspase recruitment domain contributes to chemotherapy resistance by abolishing mitochondrial fission mediated by dynamin-related protein-1. Cancer Res. 2009;69:492-500

13. Zhao J, Zhang J, Yu M, et al. Mitochondrial dynamics regulates migration and invasion of breast cancer cells. Oncogene. 2013;32(40):4814 4824. Epub 2012 Nov 5

14. Han XJ, Yang ZJ, Jiang LP, et al. Mitochondrial dynamics regulates hypoxia-induced migration and antineoplastic activity of cisplatin in breast cancer cells. Int J Oncol. 2015;46:691-700.

15. Wiznerowicz M, Trono, D. Conditional suppression of cellular genes: lentivirus vector-mediated drug-inducible RNA interference. $J$ Virol. 2003;77(16):8957-8961.

16. Gao M, Wang J, Wang W, Liu J, Wong CW. Phosphatidylinositol 3-kinase affects mitochondrial function in part through inducing peroxisome proliferator-activated receptor gamma coactivator-1beta expression. Br J Pharmacol. 2011;162:1000-1008.

17. Goldstein JC, Waterhouse NJ, Juin P, Evan GI, Green DR. The coordinate release of cytochrome $\mathrm{c}$ during apoptosis is rapid, complete and kinetically invariant. Nat Cell Biol. 2000;2:156-162.

18. Boland ML, Chourasia AH, Macleod KF. Mitochondrial dysfunction in cancer. Front Oncol. 2013;3:292.

19. Grandemange S, Herzig S, Martinou JC. Mitochondrial dynamics and cancer. Semin Cancer Biol. 2009;19:50-56.

20. Chen H, Detmer SA, Ewald AJ, Griffin EE, Fraser SE, Chan DC. Mitofusins Mfn1 and Mfn2 coordinately regulate mitochondrial fusion and are essential for embryonic development. J Cell Biol. 2003;160: 189-200.

21. Stojanovski D, Koutsopoulos OS, Okamoto K, Ryan MT. Levels of human Fis1 at the mitochondrial outer membrane regulate mitochondrial morphology. J Cell Sci. 2004;117:1201-1210.

22. Diehn M, Cho RW, Lobo NA, et al. Association of reactive oxygen species levels and radioresistance in cancer stem cells. Nature. 2009; 458:780-783.

23. Röth D, Krammer PH, Gulow K. Dynamin related protein 1-dependent mitochondrial fission regulates oxidative signalling in T cells. FEBS Lett. 2014;588:1749-1754.

24. Zhang S, Ong CN, Shen HM. Critical roles of intracellular thiols and calcium in parthenolide-induced apoptosis in human colorectal cancer cells. Cancer Letters. 2004;208(2):143-153.

25. Wen J, You KR, Lee SY, Song CH, Kim DG. Oxidative stress-mediated apoptosis. The anticancer effect of the sesquiterpene lactone parthenolide. J Biol Chem. 2002;277(41):38954-38964.

\section{Dovepress}

patient perspectives such as quality of life, adherence and satisfaction. The manuscript management system is completely online and includes a very quick and fair peer-review system, which is all easy to use. Visit http://www.dovepress.com/testimonials.php to read real quotes from published authors. 